\title{
Implementation of an Impedance Spectroscopy Equipment for the Measurement of Electrochemical Parameters
}

\author{
O. Duran-Avendaño ${ }^{\# 1}$, C. Ramírez-Martín ${ }^{* 2}$, O. Téquita ${ }^{* 3}$, D. Figueredo $^{* 4}$, N.J. Cavieles ${ }^{+5}$ \\ * Physics School, Universidad Pedagógica y Tecnológica de Colombia, 150003 Tunja, Colombia \\ 1 oscar.duran@uptc.edu.co \\ *Physics Department, Universidad de Boyacá, 150003 Tunja, Colombia \\ 2 Corresponding Author's: cramirezm@uniboyaca.edu.co \\ ${ }^{3}$ oftequita@uniboyaca.edu.co \\ ${ }^{4}$ dfigueredo@uniboyaca.edu.co \\ + Mechatronics Engineering, Universidad de Boyacá, 150003 Tunja, Colombia \\ ${ }^{5}$ njcavieles@uniboyaca.edu.co
}

\begin{abstract}
A device for the measurement of electrochemical parameters was designed and built using the NI USB 6250 card and the LabView 2010 software. The implemented technique, Electrochemical Impedance Spectroscopy (EIS), requires the measurement of the impedance in a wide scan of frequency, as well as the generation of its corresponding Nyquist diagram. The developed equipment, allows to recognize the type of interfaces in the layers between metals and electrolytes in order to measure corrosion parameters such as the electrical resistance of the layers, together with their capacitance and the physical model of the interface.
\end{abstract}

Keyword - LabView; Nyquist; EIS; electrochemistry; Lock-in amplifier.

\section{INTRODUCTION}

When a metal is immersed in an aqueous solution and this solution has electrolytes, they form on the surface an interface where they are organized on its surface; From here a first model named double layer of HelmholtzPerrin arises in 1879 which is based on the accumulation of charges forming an equivalent capacitor and an electric resistance [1].

Subsequently, this layer was studied by (Gouy and Chapman 1910-1913) from where it is diffuse behavior was deduced in terms of the physicochemical variables of the electrolyte [2]. Currently the model are adopted between the electrolyte-metal is the Gouy-Chapman-Stern [3] that combines the two previous models as shown in Fig. 1.

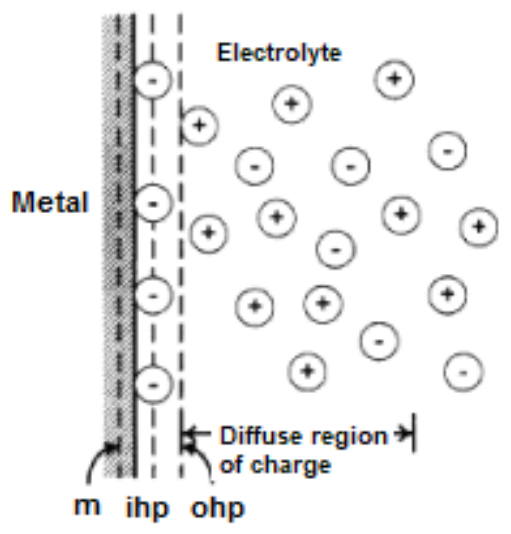

Fig. 1. Helmholtz-Perrin model+Gouy-Chapman model = Gouy-Chapman-Stern model [3].

The Helmholtz model [4] is supported as if on the surface there is a parallel plate capacitor, in the region inside the capacitor applies the Laplace equation given by

$$
\frac{\partial^{2} \phi}{\partial x^{2}}=-\frac{\rho}{\varepsilon}=0 \rightarrow \frac{\partial \phi}{\partial x}=C t
$$


where $\phi$ is the potential between the metal and the outer layer of ions (ohp), $x$ is the frontier distance of the interface, $\rho$ is the charge density and $\varepsilon$ is the electrical permittivity within the layer.

The charge density is considered to be zero in the same way as the internal layer of Helmholtz (ihp), causing the behavior of the potential in this region to vary linearly with the distance as Eq. (2):

$$
\phi_{(x)}=\phi_{e}\left(1-\frac{x}{d}\right)
$$

This model does not take into account the electrolyte ions and excludes their contribution to the behavior of potential and capacitance in the double layer. Gouy and Chapman proposed a model taking into account the ions immersed in the electrolyte, based on the charge density that depends on the ionic concentration and the charge density of these ions can be written as

$$
\rho=\sum_{i} Z_{i} F C_{i}
$$

where $Z_{i}$ is the net charge of each ion, $F$ is the Faraday constant $(96352 \mathrm{C} / \mathrm{mol}), C_{i}$ is the concentration $(\mathrm{mol} / \mathrm{L})$ of the ith species of the electrolyte.

Considering that the charge density depends on the concentration as well as the energy of the ions immersed in the solution, they respond to the Boltzman distribution [5] as shown in Eq. (4):

$$
C_{i}=C_{i(x=\infty)} e^{\left(\frac{-Z_{i} F \phi}{R T}\right)}
$$

where $R$ is the gas constant $(8.3144 \mathrm{~J} / \mathrm{K} \mathrm{mol}), e$ is the charge of the electron $\left(-1.6 \times 19^{-19} \mathrm{C}\right)$.

Using both equations $3 \& 4$ and in turn in the Poisson equation Eq. (1), is obtained the model based on the Poisson-Boltzman equation given by [6]

$$
\frac{\partial^{2} \phi}{\partial x^{2}}=-\frac{1}{\varepsilon} \sum_{i} Z_{i} F C_{\infty} e^{\left(\frac{-Z_{i} F \phi}{R T}\right)}
$$
(6):

The solution of the Eq. (5), results in (replacing the variable surface charge density $\left.\sigma=-(\varepsilon / 4 \pi)(\partial \phi / \partial \mathrm{x})_{\mathrm{x}=0}\right) \mathrm{Eq}$.

$$
\sigma= \pm\left(\frac{R T \varepsilon}{2 \pi}\right)^{1 / 2}\left[\sum_{i} C_{i}\left[e^{\left(\frac{-Z_{i} F \phi}{R T}\right)}-1\right]\right]^{1 / 2}
$$

If the ion distribution is homogeneous and symmetrical on its surface, the charge distribution behaves as follows

$$
\sigma=\left(\frac{2 R T \varepsilon}{\pi}\right)^{1 / 2} \sqrt{C \operatorname{Sinh}}\left(\frac{-Z_{i} F \phi}{2 R T}\right)
$$

This model predicts a capacitance in the double layer of Helmholtz but takes into account the diffuse contribution of the ions inside the electrolyte since this capacitance per unit area is $C_{G}=\left(\frac{\partial \sigma}{\partial \phi}\right)$ as shown in Eq. (8):

$$
C_{G}=\left(\frac{Z^{2} F^{2} \varepsilon}{2 \pi R T}\right)^{1 / 2} \sqrt{C} \operatorname{Cosh}\left(\frac{Z_{i} F \phi}{2 R T}\right)
$$

This predicts a capacitance (minimum capacitance) at a potential $\phi$ called Guouy-Champan capacity, this originates in the charge accumulation of all the electrolyte up to the surface of the metal given by [9]

$$
C_{G(\min )}=Z F\left(\frac{\varepsilon}{2 \pi R T}\right)^{1 / 2} \sqrt{C}
$$


The Stern model [7] adjusts these two models so that it is condensed assuming a net capacitance in the interface Metal-Solution which is defined as follows

$$
\frac{1}{C_{d}}=\frac{1}{C_{m-2}}+\frac{1}{C_{2-s}}=\frac{1}{C_{H}}+\frac{1}{C_{G}}
$$

where $C_{d}$ is the capacitance in the double layer.

Finally, the interface behaves as a capacitor which allows predicting parameters in the double layer as seen in Fig. 2.

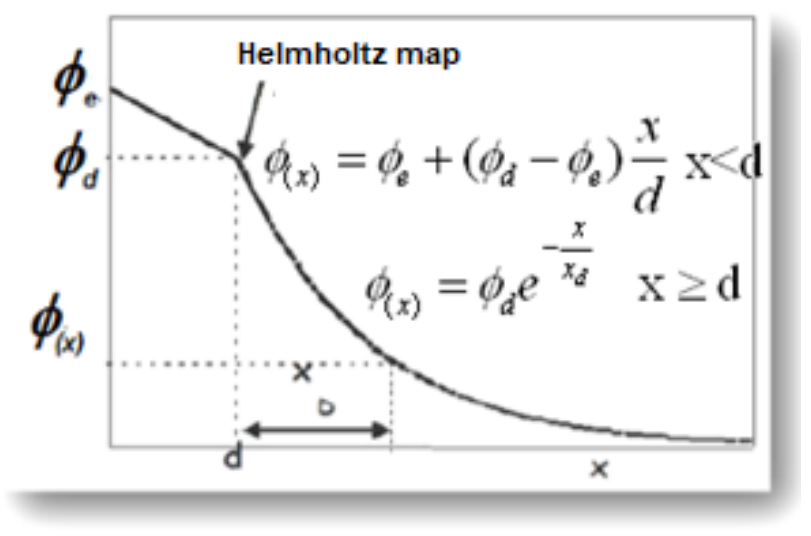

Fig. 2. Potential $\phi$ in the Stern=Gouy-Chapman+Helmholtz model.

\section{EXPERIMENTAL DEVELOPMENT}

The equipment was developed in two stages, the first focused on the hardware which consists of the analog circuit as shown in figure 3, and the second stage was developed around data acquisition and software development, taking as a reference to the National Instruments NI-USB-6250 card in charge of supplying the signal in the electrochemical cell, measuring the current signals and measuring the applied voltage. The software designed in Labview 2010 is responsible for processing the signals in order to extract the necessary data for the electrochemical characterization.

\section{A. Potentiostat Analog Circuit}

This circuit was designed based on the model commonly used in this type of equipment [3], in the plane of the circuit shown in Fig. 3.

The equipment works as follows; an AC signal from the $\mathrm{DA}_{1}$ (Digital Analogue 1) port of the NI-USB6250 card is supplied on $\mathrm{R}_{3}$; the amplifier $\mathrm{A}_{1}$ is responsible for supplying the current demanded by the cell through the counter electrode; the signal of $R_{3}$ is brought by $R_{2}$ to the voltage tracker $A_{2}$ which is responsible for driving the reference electrode $\left(\mathrm{R}_{1}\right.$ fulfills the function of increasing the impedance of this amplifier).

By means of amplifier $\mathrm{A}_{5}$, the voltage signal of the reference electrode is fed into the $\mathrm{AD}_{2}($ Digital Analog 2) port of the card; the excitation current of the cell passes through the working electrode (E. work) through the amplifier $A_{3}$ which performs the function of the current-to-voltage converter; $R_{5}$ fulfills the function of converting the current into voltage; $\mathrm{R}_{5}$ is a resistor that is selected by a mini relay where there are resistors that have the following value according to their selection $10 \Omega, 100 \Omega, 1 \mathrm{k} \Omega, 10 \mathrm{k} \Omega, 100 \mathrm{k} \Omega$ and $1 \mathrm{M} \Omega ; \mathrm{R}_{4}$ fulfills the function of increasing the impedance of $\mathrm{A}_{3}$ in order to avoid deviations of the current to be measured according to the Eq. (11): 

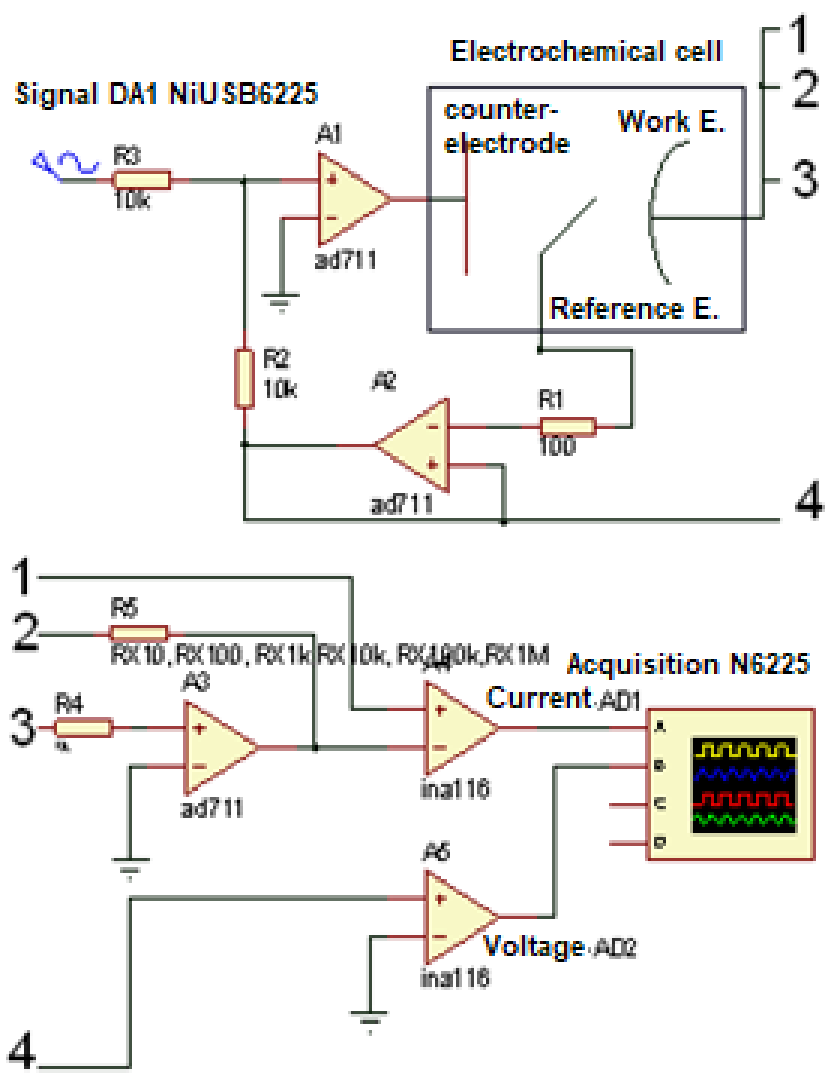

Fig. 3. EIS potentiostat analog circuit

$$
\begin{aligned}
& I_{D C x}=I_{\text {Input }} \operatorname{Cos}\left(\omega t+\phi_{i}\right) \operatorname{Cos}(\omega t) \Rightarrow \overline{I_{D C x}}=\frac{1}{2} I_{\text {Input }} \operatorname{Cos}\left(\phi_{i}\right) \\
& I_{D C y}=I_{\text {Input }} \operatorname{Cos}\left(\omega t+\phi_{i}\right) \operatorname{Sin}(\omega t) \Rightarrow \overline{I_{D C y}}=\frac{1}{2} I_{\text {Input }} \operatorname{Sin}\left(\phi_{i}\right) \\
& V_{D C x}=V_{\text {Input }} \operatorname{Cos}\left(\omega t+\phi_{i}\right) \operatorname{Cos}(\omega t) \Rightarrow \overline{V_{D C x}}=\frac{1}{2} V_{\text {Input }} \operatorname{Cos}\left(\phi_{i}\right) \\
& V_{D C y}=V_{\text {Input }} \operatorname{Cos}\left(\omega t+\phi_{i}\right) \operatorname{Sin}(\omega t) \Rightarrow \overline{V_{D C y}}=\frac{1}{2} V_{\text {Input }} \operatorname{Sin}\left(\phi_{i}\right)
\end{aligned}
$$

Once the $\mathrm{AD}_{1}$ and $\mathrm{AD}_{2}$ voltages have been acquired, the measure of the amplitude and phase from the electrochemical cell is made by the subVI (lock-in) where they are each multiplied by two reference signals having the same amplitude and frequency at the excitation but phase-shifted $90^{\circ}$, between them according to Fig. 4.

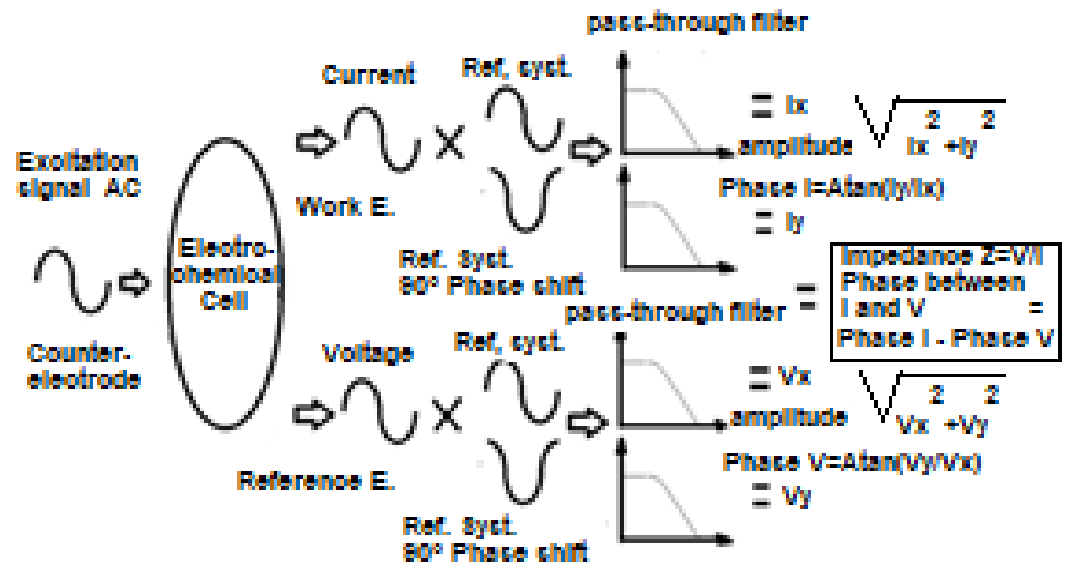

Fig. 4. Lock-In technique (phase-sensitive detection) 
After being multiplied by the reference signal each one goes through a low-pass filter to obtain a $D C$ signal $\left(I_{D C x}, I_{D C y}, V_{D C x}, V_{D C y}\right)$ representing the amplitude of the measured signal; since the original signal was multiplied by two out-of-phase signals each one is used to obtain the phase with respect to the reference signal [8-10].

From Eq. (11) the components of the current and voltage as well as the phases of each are obtained with respect to the reference signal as shown in Eq. (12):

$$
\begin{aligned}
& I_{\text {Input }}=\sqrt{2 \sqrt{I_{D C x}}{ }^{2}+{\overline{I_{D C y}}}^{2}} ; \quad \phi_{i}=A \tan \left(\frac{\overline{I_{D C y}}}{\bar{I}_{D C x}}\right)
\end{aligned}
$$

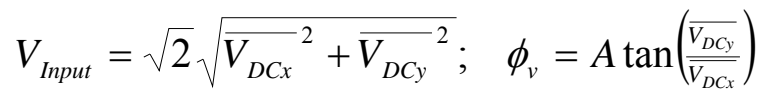

Using Eq. (12) gives the Eq. (13) and Eq. (14) to implement the impedance spectra.

$$
\begin{aligned}
& Z_{(\omega)}=\frac{V_{\text {input }}}{I_{\text {input }}} \\
& \phi_{i-v}=\phi_{i}-\phi_{v}
\end{aligned}
$$

\section{III.RESULTS}

The verification of the correct functioning of the equipment was performed by measuring different spectra in emulated conditions (dummy circuits) and simulated (with Zsimpwin). For the validation of the data we analyzed the theoretical models realized in the program Zsimpwin version 3.20 developed by Ph.D. Bruno Yeum [11], this version of the software was used thanks to its precision in the analysis of electrochemical models.

\section{A. Circuit 1}

The cicuit of Fig. 5, emulates double layer behavior with passivating oxide coating (typical characteristic values according to bibliography) [12], the measured spectrum is observed in the Fig. 6 and the simulation in Zsimpwin is represented in the Fig. 7

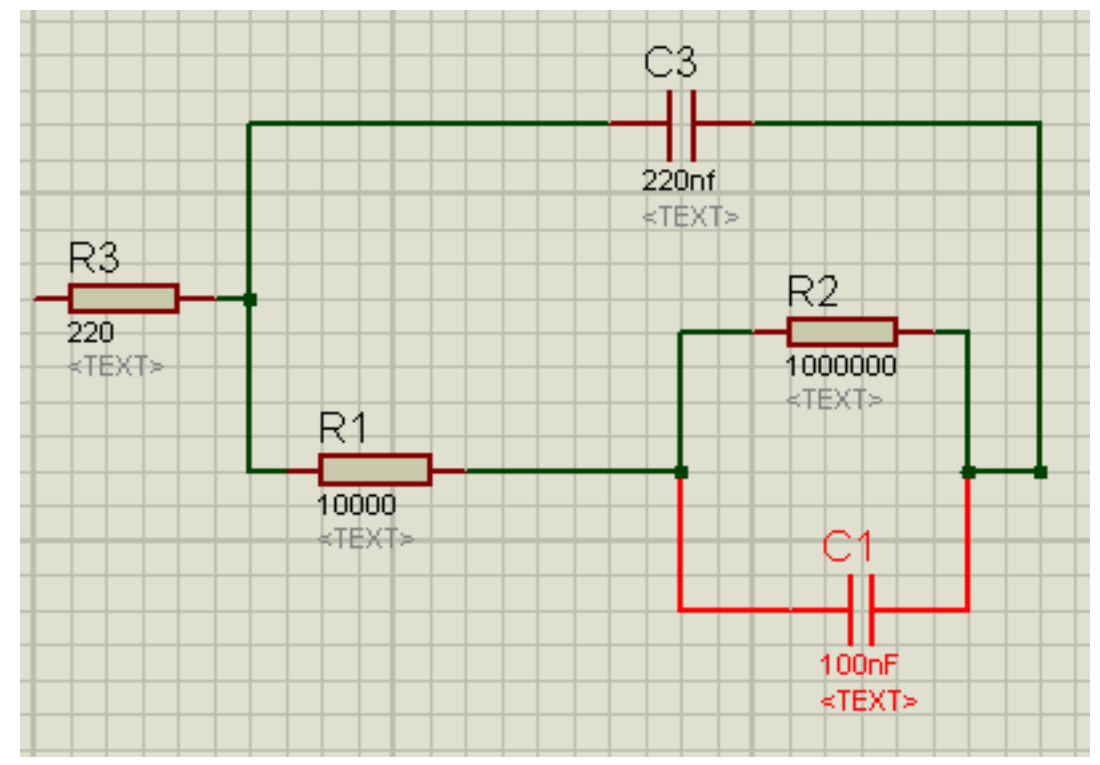

Fig. 5. Dummy cicuits 1 


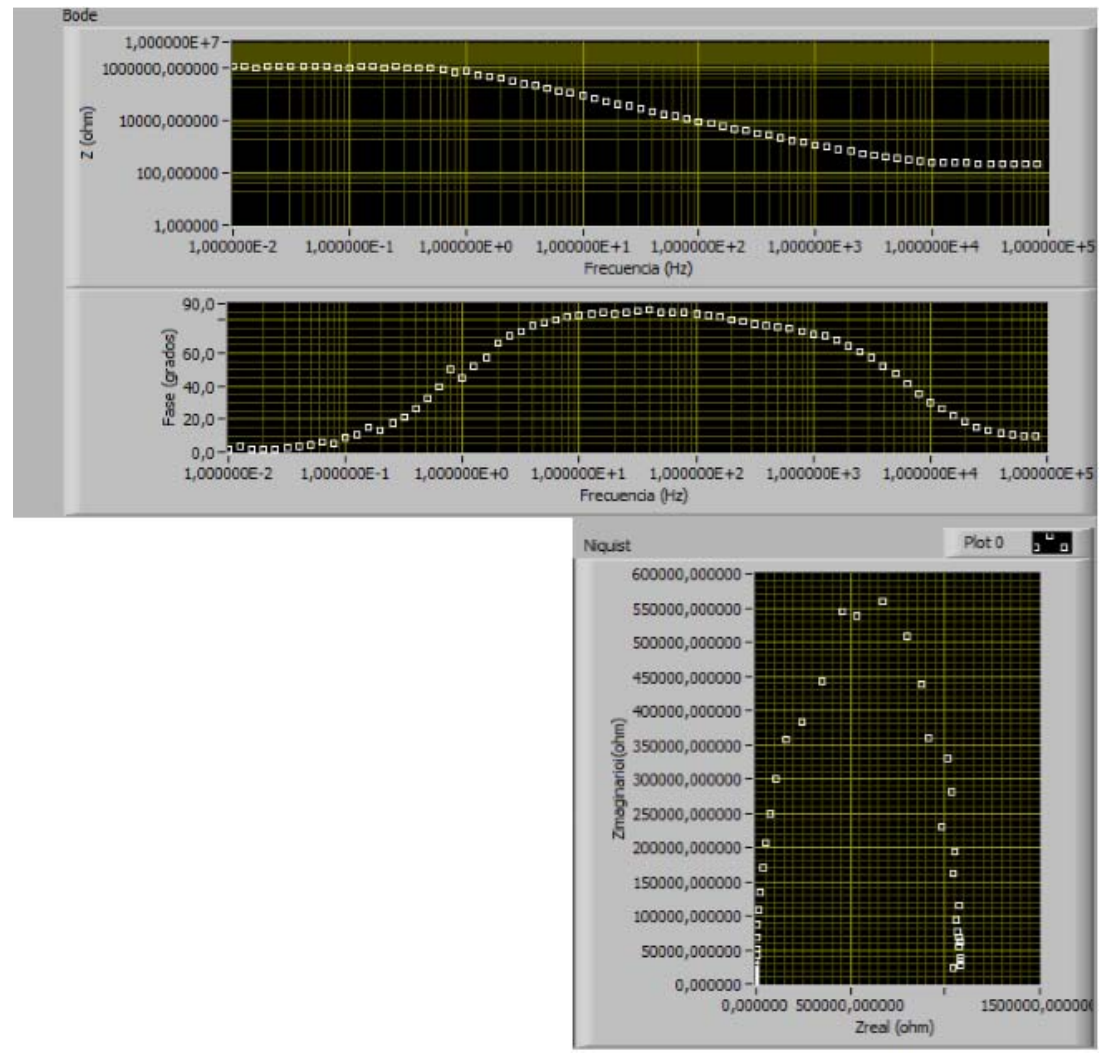

Fig. 6. Circuit spectrum dummy 1 taken by the equipment
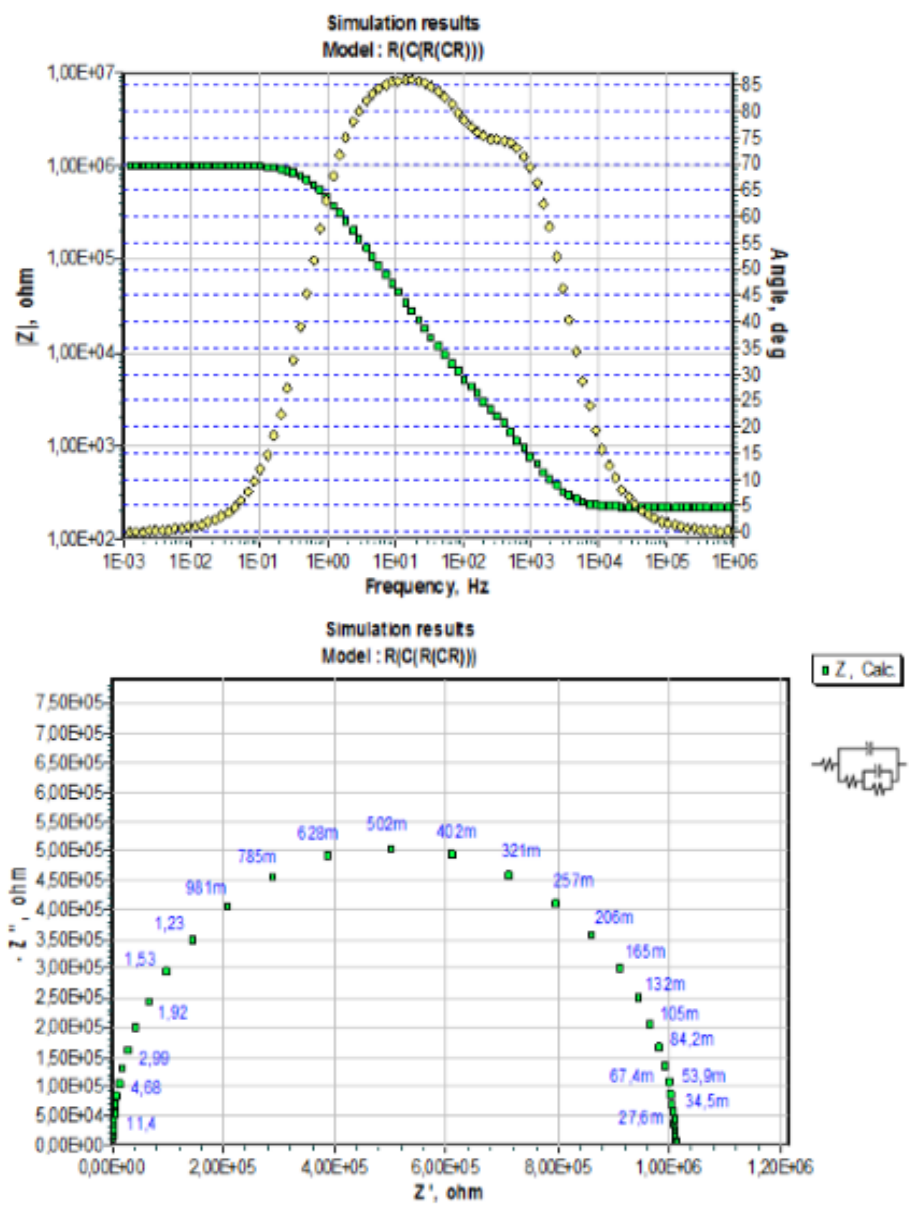

Fig. 7. Circuit spectrum dummy 1 simulated in Zsimpwin 
ISSN (Print) : 2319-8613

\section{B. Circuit 11}

The dummy circuit 2 shown in Fig. 8 which does not emulate specific electrochemical surface condition was tested and measured as shown in Fig. 9 in order to compare its impedance spectrum and the simulation in Zsimpwin shown in Fig. 10.

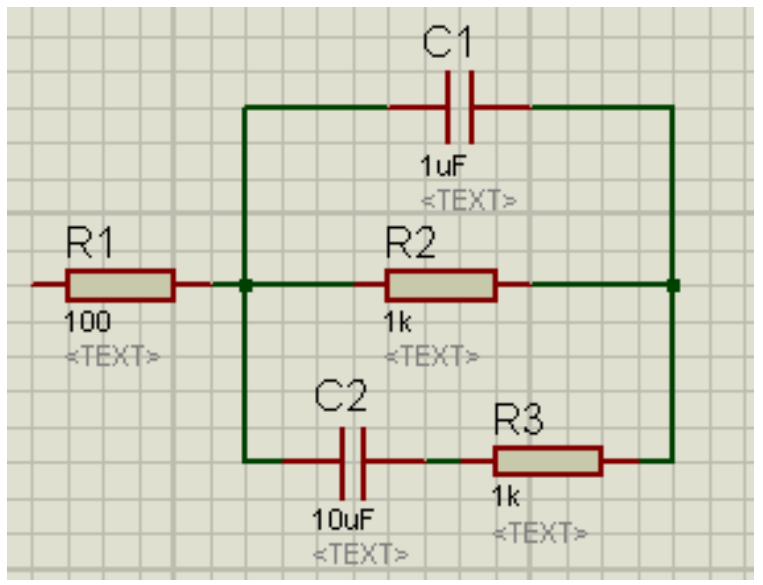

Fig. 8. Circuit dummy 2
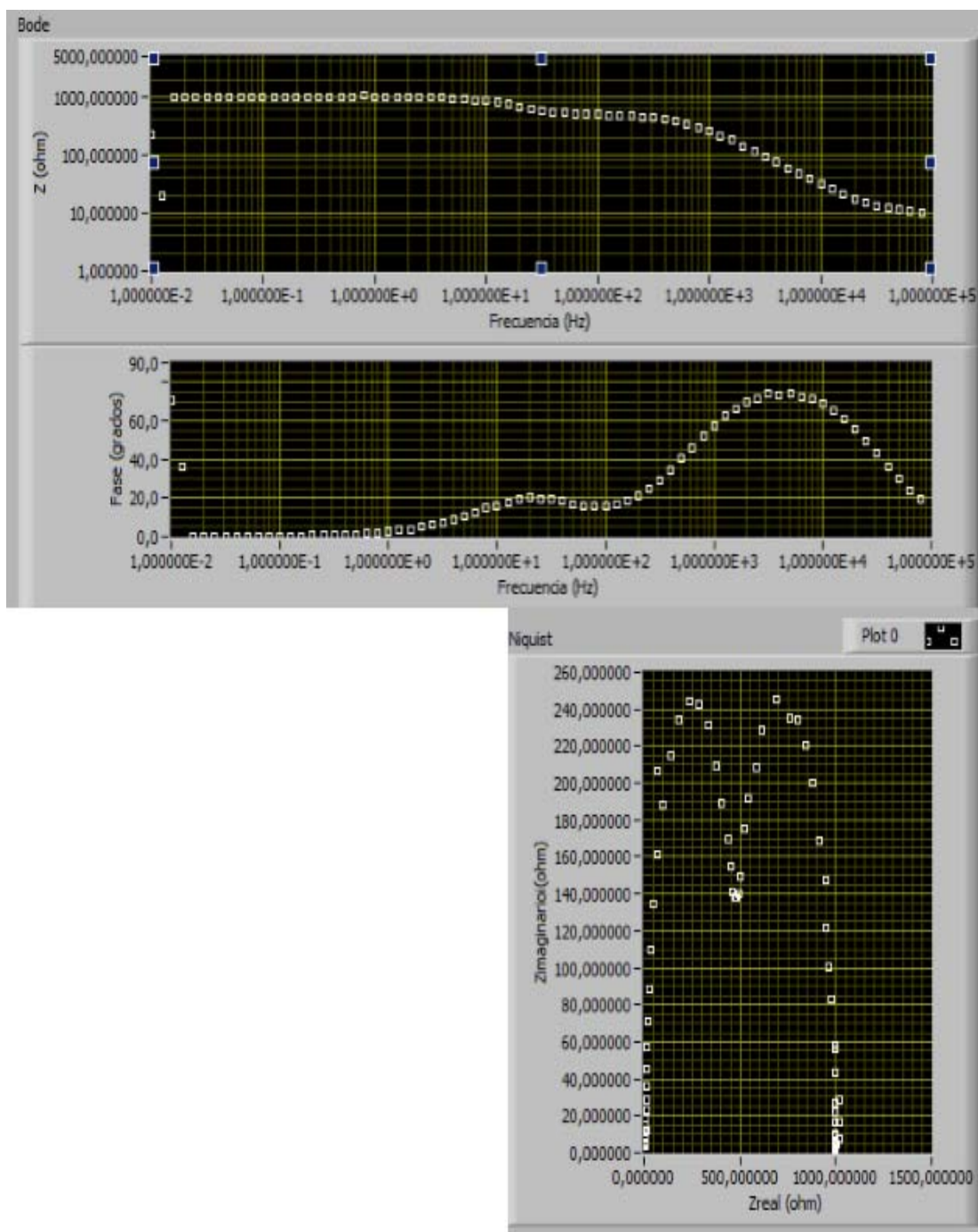

Fig. 9. Circuit spectrum dummy 2 taken by the equipment 

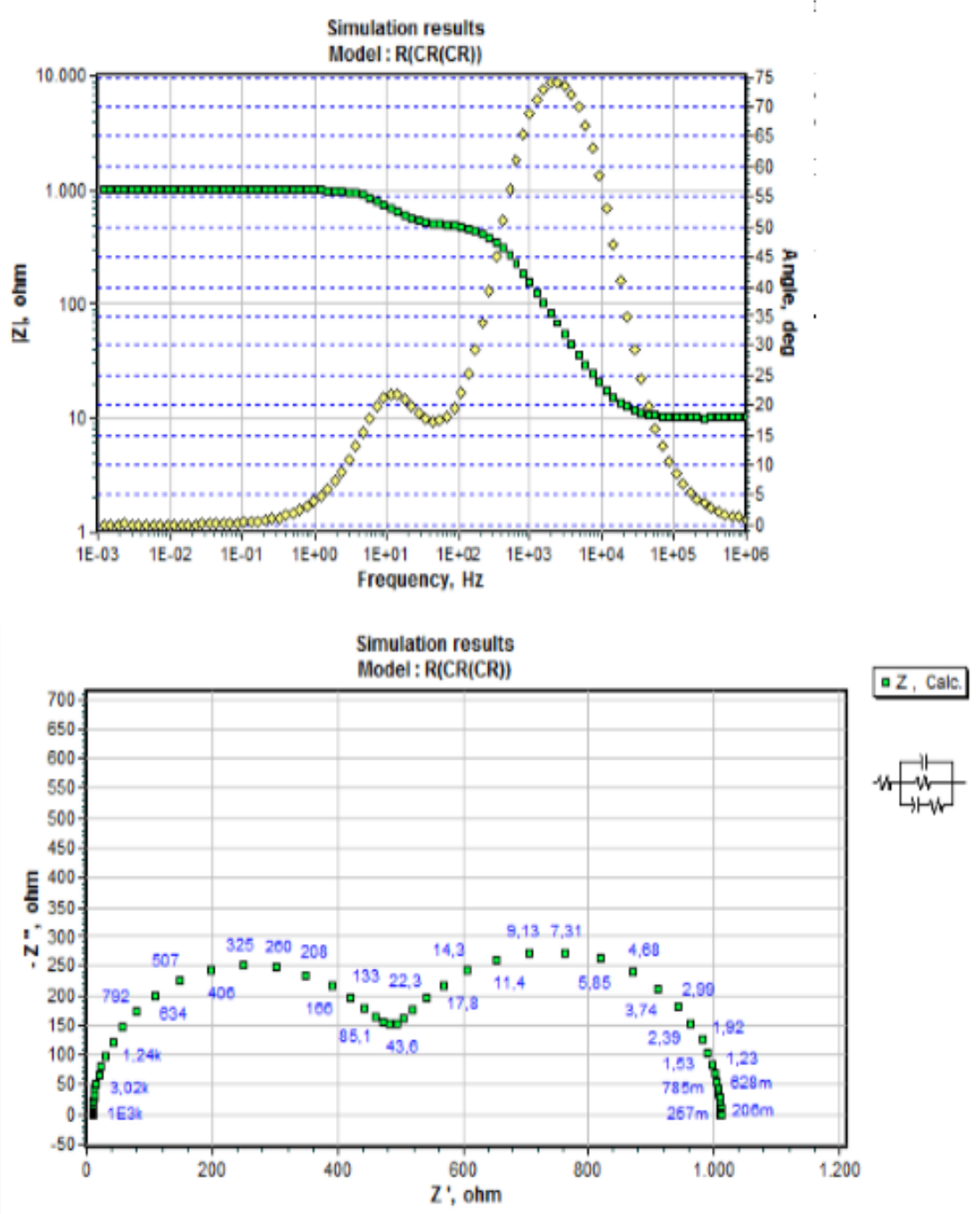

Fig. 10. Spectrum circuit dummy 2 simulated in Zsimpwin

\section{Electrochemical Cell}

In the electrochemical cell, $316 \mathrm{~L}$ steel and $10 \%$ sulfuric acid were used as the electrolyte; the measurement parameters were: $\mathrm{V}_{\text {offset }}=0 \mathrm{~V}$, reference electrode $\mathrm{V}=10 \mathrm{mV}, \mathrm{f}_{\text {initial }}=0.01 \mathrm{~Hz}, \mathrm{f}_{\text {final }}=100 \mathrm{kHz}$, frequency resolution $=10$ data per decade. In the spectrum observed on the cell, electric noise is pronounced, especially at low frequencies, as shown in Fig. 11.

The spectrum measured by the equipment coincides with the typical double layer model [8] where the resistance of the measured solution $\left(R_{s}\right)$ was $240 \Omega$, this is justified in the fact that the sulfuric acid used in this concentration has a high ionic conductivity.

A double layer formation with a capacitance formed by the passive layer $\left(\mathrm{C}_{\mathrm{p}}\right)$ generated by the chromium $\left(\mathrm{Cr}_{2} \mathrm{O}_{3}\right)$ oxides as well as the Helmholtz $(\mathrm{Ch})$ capacitance on the surface was also observed, the passive layer obtained measured approximately $0.8 \mu \mathrm{F}$ and the Helmholtz layer showed an approximate capacity of $4.5 \mathrm{nF}$, resulting in a medium accumulation of protons in the Helmholtz layer and in the polarization of the passive layer. 


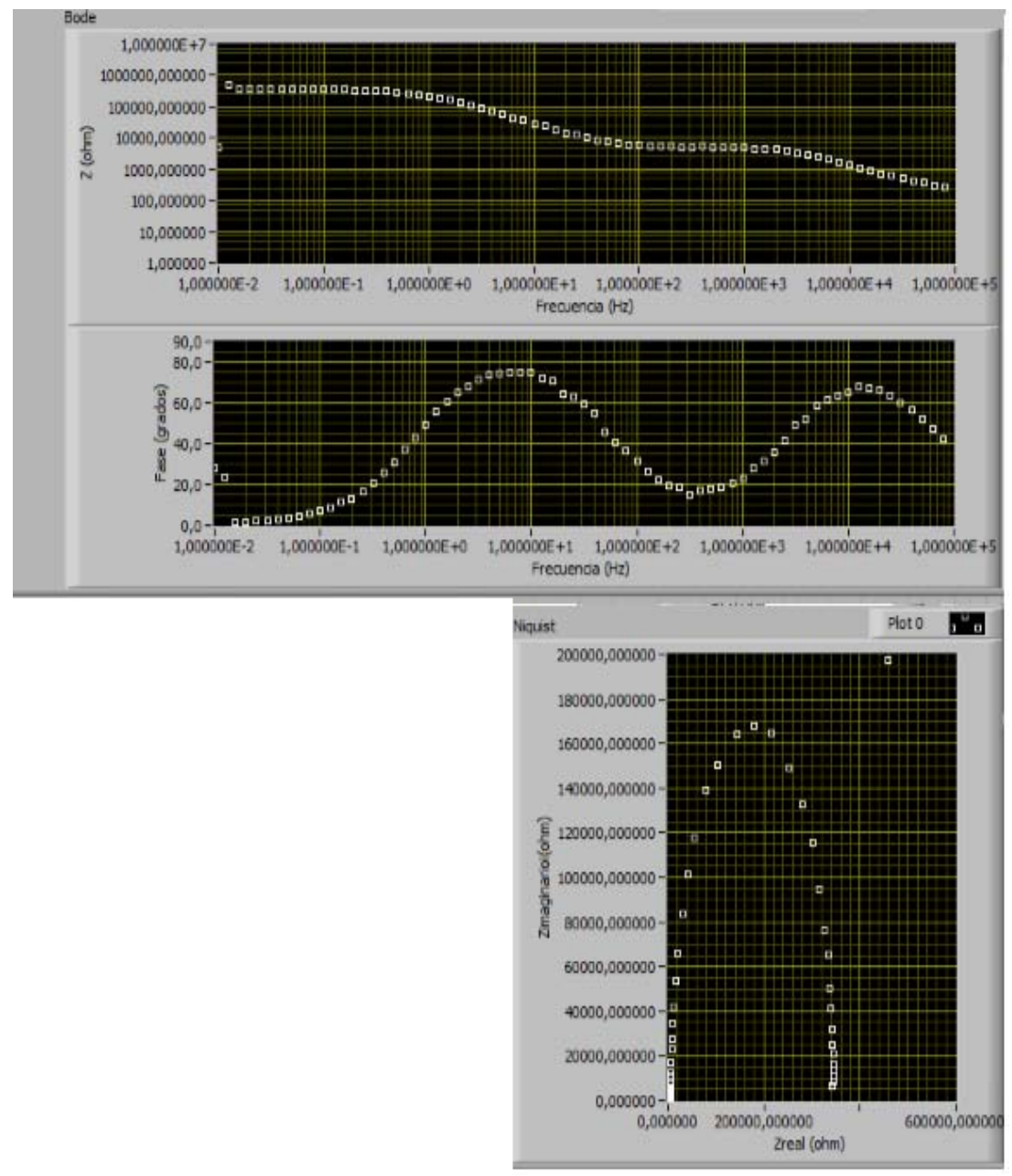

Figure 11. Spectrum of the electrochemical cell Steel $316 \mathrm{~L}$, in $10 \% \mathrm{H}_{2} \mathrm{SO}_{4}$

The impedance of the passive layer $\left(\mathrm{R}_{\mathrm{p}}\right)$ was approximately $340 \mathrm{k} \Omega$ while the impedance of the Helmholtz $\left(R_{h}\right)$ layer was in the order of $4700 \Omega$ coinciding with the fact that it was a stainless steel. This layer generated on the steel surface coincides of being highly insulating (almost impenetrable by the ions). Although this layer is not indestructible it is restored to continue to protect the metal each time it is broken [10;13-15]. The typical model of this layer are shown in Fig. 12, which was simulated in Zsimpwin and is observed in Fig. 13.

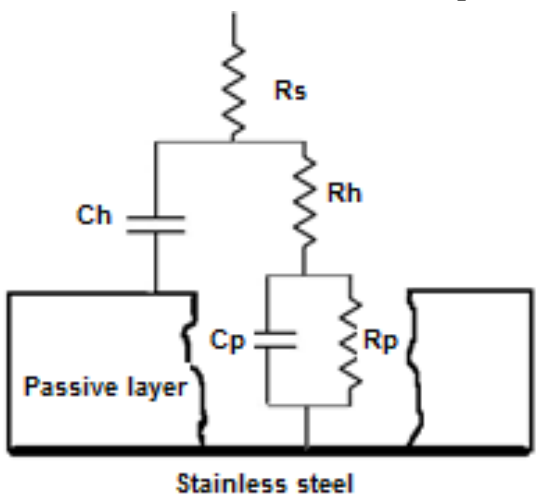

Fig. 12. Double-layer model in 316L stainless Steel

The electrical resistance values of the bilayer were obtained from the impedance measurement in the frequency spectrum of Fig. 11. For frequencies below $0.1 \mathrm{~Hz}$ the impedance is approximately constant and is maintained at a value of $340 \mathrm{~K} \Omega$, in the range of $100 \mathrm{~Hz}$ to $1 \mathrm{kHz}$ the value returns to approximately constant of $4700 \Omega$, from $2 \mathrm{kHz}$ the impedance falls again until it reaches a nearly constant value near $90 \mathrm{k} \Omega$; these three constant impedances are the impedances of the first layer $R_{p}$, the second layer $R_{h}$ and the resistance of the solution $\mathrm{R}_{\mathrm{s}}$ according to the model of Fig. 12. 

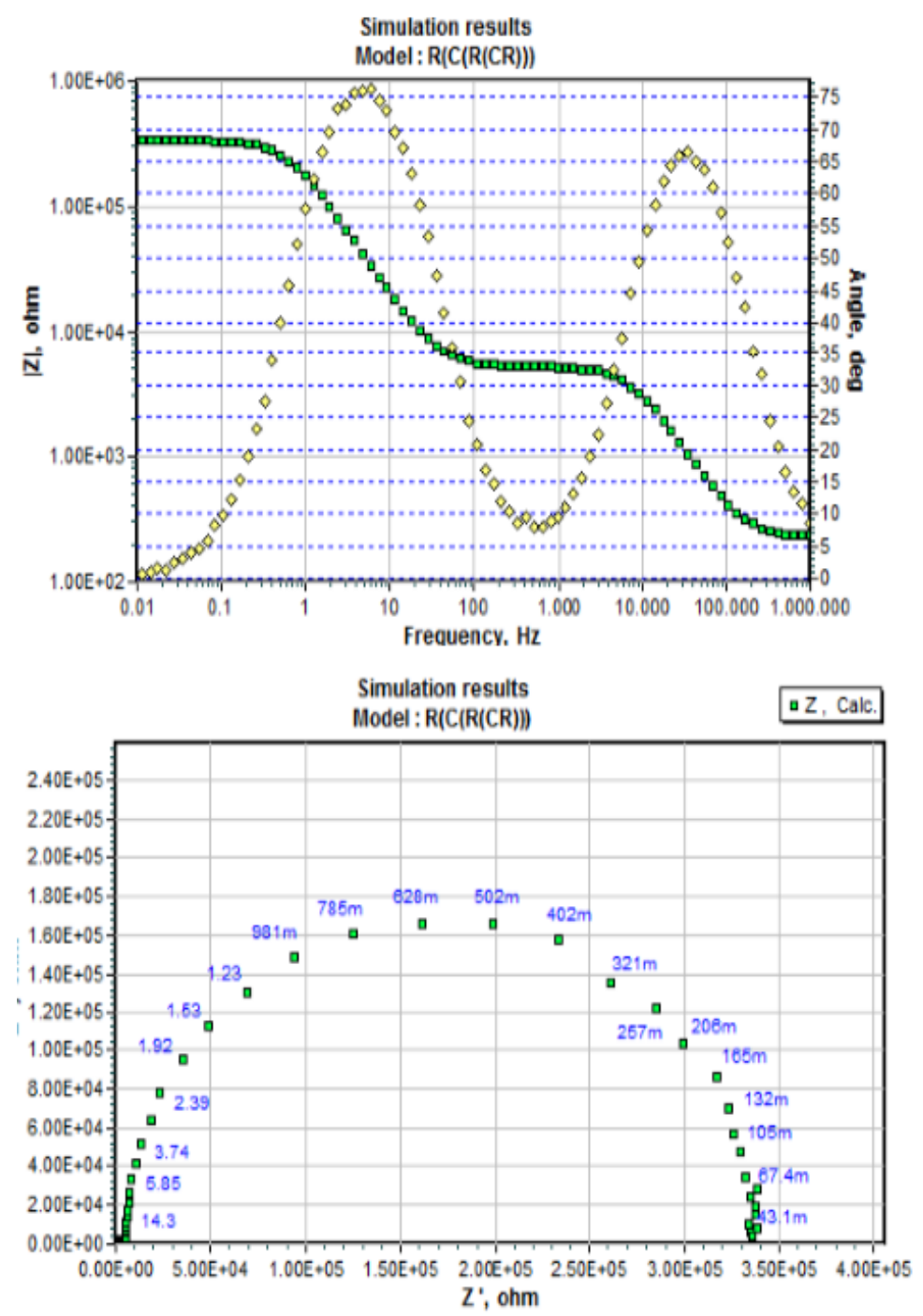

Fig. 13. Simulation of the model according to figure 12 of the electrochemical cell in Zsimpwin

Two capacitances were observed from the diagrams due to two notorious bumps in the phase versus frequency located at $7 \mathrm{~Hz}$ and $17 \mathrm{kHz}$ approximately, these frequencies are determinant for the calculation of capacitances $C_{h}$ and $C_{p}$ of the model used which were calculated from the Following form.

For the calculation of $C_{p}$, a maximum of phase change at $17 \mathrm{kHz}$ is observed for the complex impedance (capacitive); at this frequency the complex impedance has a value close to $2 \mathrm{k} \Omega$; these values allow to calculate the capacitance $C_{p}$ according to the relation $\mathrm{C}=(2 \pi \mathrm{fZ})^{-1}$ [2], replacing these values results in an approximate value of $4.5 \mathrm{nF}$.

By performing the same procedure for the calculation of $C_{\mathrm{h}}$, it is observed that the maximum phase change for this capacitance is around $7 \mathrm{~Hz}$, having a complex impedance value close to $25 \mathrm{k} \Omega$; by replacing in the relation $\mathrm{C}=(2 \pi \mathrm{fZ})^{-1}$ results in a value of $0.8 \mu \mathrm{F}$ for $C_{h}$. The impedance values allow to appreciate the conductive tendency of the layer, as it is for the case of $316 \mathrm{~L}$ stainless steel.

While the capacitance allows us to perceive the amount of charge accumulated in the interface allowing to postulate a model around the Bode and Nyquist diagrams. In order to validate the data in statistical form, the Zsimwin 3.20 program was used, which has the option to load the data of frequency, real and complex impedance in any file with .txt extension and to perform the statistical procedure based on the transforms of Kramers-Kronig [16].

This program gives a result in terms of $\chi^{2}$ which is the final validation parameter of the equipment; the calculation of the values of $\chi^{2}$ for each dummy circuit and for the electrochemical cell yielded the following results, circuit $1 \chi^{2}=3.4 \times 10^{-6}$, for circuit $2 \chi^{2}=7.5 \times 10^{-7}$, for the cell electrochemistry $\chi^{2}=1.2 \times 10^{-5}$, and in other test circuits the values $\chi^{2}$ gave similar results, to these data checked in a quantitative form using the KramersKronig transforms [14], that measurements are in the range of acceptable and good. 


\section{CONCLUSION}

An electrochemical impedance spectroscopy equipment was designed and built based on the National Instruments NI 6250 data acquisition card and the Labview G-language program. The operation of the equipment was based on measurements made by commercial teams whose objective is to obtain the Bode and Nyquist diagrams for analysis. The equipment has a measurement range of $0.01 \mathrm{kHz}$ up to $100 \mathrm{kHz}$ in bandwidth, measures impedances from $0.1 \Omega$ to $10 \mathrm{M} \Omega$; has the ability to vary voltages at the reference of electrode from $2 \mathrm{mV}$ to $400 \mathrm{mV}$ amplitude.

The measurements are performed using the Lock-in amplifier technique programmed in a subVI of the program which are allow to obtain measurements of high precision in amplitude and phase shift in AC signals in the presence of electrical noise. The program consists of a general VI (virtual instrument) which is supported by several subVI's each with its corresponding function. The advantage of the equipment lies in the simplicity of its hardware and software, which can make viable its easy commercialization and debugging.

In order to verify the correct functioning of the equipment and the validation of its data, tests were carried out on different Dummy circuits characterized in real electrochemical interfaces and compared with the simulations made by the program Zsimpwin 3.20, a test was also carried out in an electrochemical cell with 316L steel immersed in $\mathrm{H}_{2} \mathrm{SO}_{4}$ obtaining consistent results.

\section{REFERENCES}

[1] S. Grimnes and O. Grottem, Bioimpedance and Biolelectricity, London; England: Academic Press, 2005

[2] J. M. Bockris; A.K.N. Reddy, Modern Electrochemistry, vol. 1, Madrid, Spain: Reverté, 1978.

[3] J. M. Bockris; A.K.N. Reddy, Modern Electrochemistry, vol. 2, Madrid, Spain: Reverté, 1980.

[4] H. B. Sierra-Alcazar, A. N. Fleming and J. A. Harrison, "An Assessment of the measurements of impedance by analysis of a pulse using an on-line computer," Journal of Electroanalytical Chemistry, vol. 87, no.3, pp.339-345, March 1997.

[5] M. Rueda and F. Prieto, "Application of electrochemical impedance spectroscopy to the study of surface processes," Electrochemical Impedance Spectroscopy of Surface, vol. 76, no.11, pp.1825-1854, January 2011.

[6] M. Kendig and F. Mansfeld, "Corrosion rates from impedance measurements: an improved aproach for rapid automatic analysis", Corrosion, vol. 39, no. 11, pp. 466-467, November 1983.

[7] Wolfgan, Interfacial electrochemistry, Oxford, England: University Press, 1996.

[8] Stanford Research Systems, "Lock-in amplifier model SR510 Inc.", November 2013.

[9] L. Andrzej, "Electrochemical Impedance Spectroscopy and its applications", New York, United States: Kluwer/Academic, 1999.

[10] (2015) METROHM-AUTOLAB website. Application note 1.5 [online]. Avalaible: http://www.metrohm-autolab.com

[11] C. Gabriell, M. Keddam, F. Huet and J.F. Lizee, "Measurement time versus accuracy trade-off analyzed for electrochemical impedance measurements by means of sine, white noise and step signals," J. Electroanal. Chem., vol. 138, no. 1, pp.201-208, August 1982.

[12] R. Antaño, "Aplicación de un algoritmo basado en un modelo de medición para la detección de errores en las medidas experimentales de impedancia," M. Eng. Thesis, Universidad Autónoma Metropolitana-Iztapalapa, División de Ciencias Básicas e Ingeniería, Iztapalapa, Mexico, January, 1997.

[13] M. Urquidi-Macdonald, S. Real and D. MacDonald, "Application of Kramers-Kronig Transforms in the Analysis of Electrochemical Impedance Data: II Transformations in the ComplexPlane," Journal of the Electrochemycal Society, vol. 133, no.10, pp.2018-2024, October 1986.

[14] I. Rubinstein, Fundamentals of physical electrochemistry, New York, United States: M. Dekker, 1995.

[15] Echen Software. (2004). ZsimpWin. [online]. Avalaible: http://www.echemsw.com/prod01.htm

[16] S. Wolfgan, Interfacial electrochemistry, Oxford, England: University Press, 1996. 